\title{
LUMINOUS COMPACT BLUE GALAXIES UP TO $z \sim 1$ IN THE HUBBLE SPACE TELESCOPE ULTRA DEEP FIELD. I. SMALL GALAXIES OR BLUE CENTERS OF MASSIVE DISKS?
}

\author{
K. G. Noeske, D. C. Koo, A. C. Phillips, C. N. A. Willmer, and J. Melbourne \\ UCO/Lick Observatory, University of California, Santa Cruz, 1156 High Street, Santa Cruz, CA 95064; kai@ucolick.org \\ A. GIL DE PAZ \\ Observatories of the Carnegie Institution of Washington, 813 Santa Barbara Street, Pasadena, CA 91101 \\ AND \\ P. PAPADEROS \\ Institut für Astrophysik, Universität Göttingen, Friedrich-Hund-Platz 1, D-37077 Göttingen, Germany \\ Received 2005 October 5; accepted 2006 February 21; published 2006 March 9
}

\begin{abstract}
We analyze 26 luminous compact blue galaxies (LCBGs) in the Hubble Space Telescope ACS Ultra Deep Field (UDF) at $z \sim 0.2-1.3$, to determine whether these truly are small galaxies or, rather, bright central starbursts within existing or forming large disk galaxies. Surface brightness profiles from UDF images reach fainter than rest-frame $26.5 B$ mag $\operatorname{arcsec}^{-2}$ even for compact objects at $z \sim 1$. Most LCBGs show a smaller, brighter component that is likely star-forming, and an extended, roughly exponential component with colors suggesting stellar ages from $\gtrsim 100 \mathrm{Myr}$ to a few gigayears. Scale lengths of the extended components are mostly $\lesssim 2 \mathrm{kpc}$, more than 1.5-2 times smaller than those of nearby large disk galaxies like the Milky Way. Larger, very low surface brightness disks can be excluded down to faint rest-frame surface brightnesses ( $\left.226 \mathrm{~B} \mathrm{mag} \operatorname{arcsec}^{-2}\right)$. However, one or two of the LCBGs are large, disklike galaxies that meet LCBG selection criteria because of a bright central nucleus, possibly a forming bulge. These results indicate that $\gtrsim 90 \%$ of high- $z$ LCBGs are small galaxies that will evolve into small disk galaxies, or low-mass spheroidal or irregular galaxies in the local universe, assuming passive evolution and no significant disk growth. The data do not reveal signs of disk formation around small, H II galaxy-like LCBGs, nor do they suggest a simple inside-out growth scenario for larger LCBGs with a disklike morphology. Irregular blue emission in distant LCBGs is relatively extended, suggesting that nebular emission lines from star-forming regions sample a major fraction of an LCBG's velocity field.
\end{abstract}

Subject headings: galaxies: compact — galaxies: evolution — galaxies: high-redshift galaxies: kinematics and dynamics — galaxies: starburst — galaxies: structure

\section{INTRODUCTION}

The term "luminous compact blue galaxies" (LCBGs) describes small, luminous $\left(\leq L_{*, B}\right)$, high surface brightness galaxies with blue optical colors and strong emission lines (Guzmán et al. 2003; Garland et al. 2004; Werk et al. 2004). Such objects had previously been classified as, for example, faint blue galaxies, compact narrow emission line galaxies (CNELGs; Koo et al. 1995; Guzmán et al. 1996), luminous compact galaxies (Hammer et al. 2001, 2005), and blue compact galaxies (Pisano et al. 2001), with varying selection criteria.

Prior work has indicated that LCBGs are progenitors of different intermediate- and low-mass galaxies in the local universe that are brightened by intense star formation (SF) (e.g., Koo et al. 1995; Guzmán et al. 1997). Galaxies undergoing an LCBG phase may contribute $\sim 45 \%$ of the comoving UV-derived SF rate density of the universe and $20 \%$ of the field galaxy number density at $z \sim 1$ (Phillips et al. 1997; Guzmán et al. 1997), and they show the strongest known number density decline $(\times 10$ 100) from intermediate $z(\sim 0.4-1)$ to $z=0$; they are almost absent in the local universe (Koo et al. 1994; Guzmán et al. 1997; Phillips et al. 1997). LCBG phases do therefore contribute sizably to the evolutionary phenomena observed in the whole galaxy population to $z=1$-the global increase of SF activity (Madau et al. 1996), and the luminosity and number density evolution of blue galaxies (Willmer et al. 2005; Faber et al. 2005).

This Letter addresses the controversial question of which types of local galaxies, or which of their subcomponents, experienced the LCBG phases of massive SF at redshifts $z \gtrless 0.2$ to $z>1$. Koo et al. (1995), Guzmán et al. (1996, 1998), and
Phillips et al. (1997) distinguished smaller LCBGs (half-light radius $\left.r_{e} \leqslant 3 \mathrm{kpc}\right)$, with low velocity dispersion $\left(\sigma_{v} \lesssim 65 \mathrm{~km}\right.$ $\mathrm{s}^{-1}$ ) and starburst dwarf-like morphologies, from larger, more massive LCBGs $\left(65 \mathrm{~km} \mathrm{~s}^{-1} \lesssim \sigma_{v} \lesssim 160 \mathrm{~km} \mathrm{~s}^{-1}\right)$, more similar to local irregular and starburst disk galaxies. They argued that the former may fade to ultimately become local low-mass spheroidal or dwarf elliptical $(\mathrm{dE})$ galaxies while the latter may evolve into local small disk and irregular galaxies. On the other hand, Hammer et al. $(2001,2005)$ and Barton \& van Zee (2001) suggested that LCBGs probably represent interaction-induced formation of bulges in today's massive spiral galaxies, possibly accompanied by inside-out disk formation (Hammer et al. 2001). In this scenario, the apparently small line widths and sizes of LCBGs do not represent intrinsic properties of these galaxies: as suggested by Koo et al. (1995) and shown by Barton \& van Zee (2001), a nuclear starburst in an ordinary extended disk can skew its effective radius, effective surface brightness, and colors to mimic a blue compact galaxy. The nuclear burst's nebular emission would sample only the inner part of the galaxy's velocity field and thus lead to an underestimate of its dynamical mass. This scenario becomes particularly worrisome with increasing redshift, where cosmological surface brightness dimming hampers the detection of low surface brightness (LSB) components and nebular emission lines are usually the only indicator of faint galaxies' kinematics.

To constrain the above scenarios, we present the first structural study of the extended components in intermediate- $z$ LCBGs, using the uniquely deep images from the Hubble Space Telescope (HST) Advanced Camera for Surveys (ACS) Ultra 

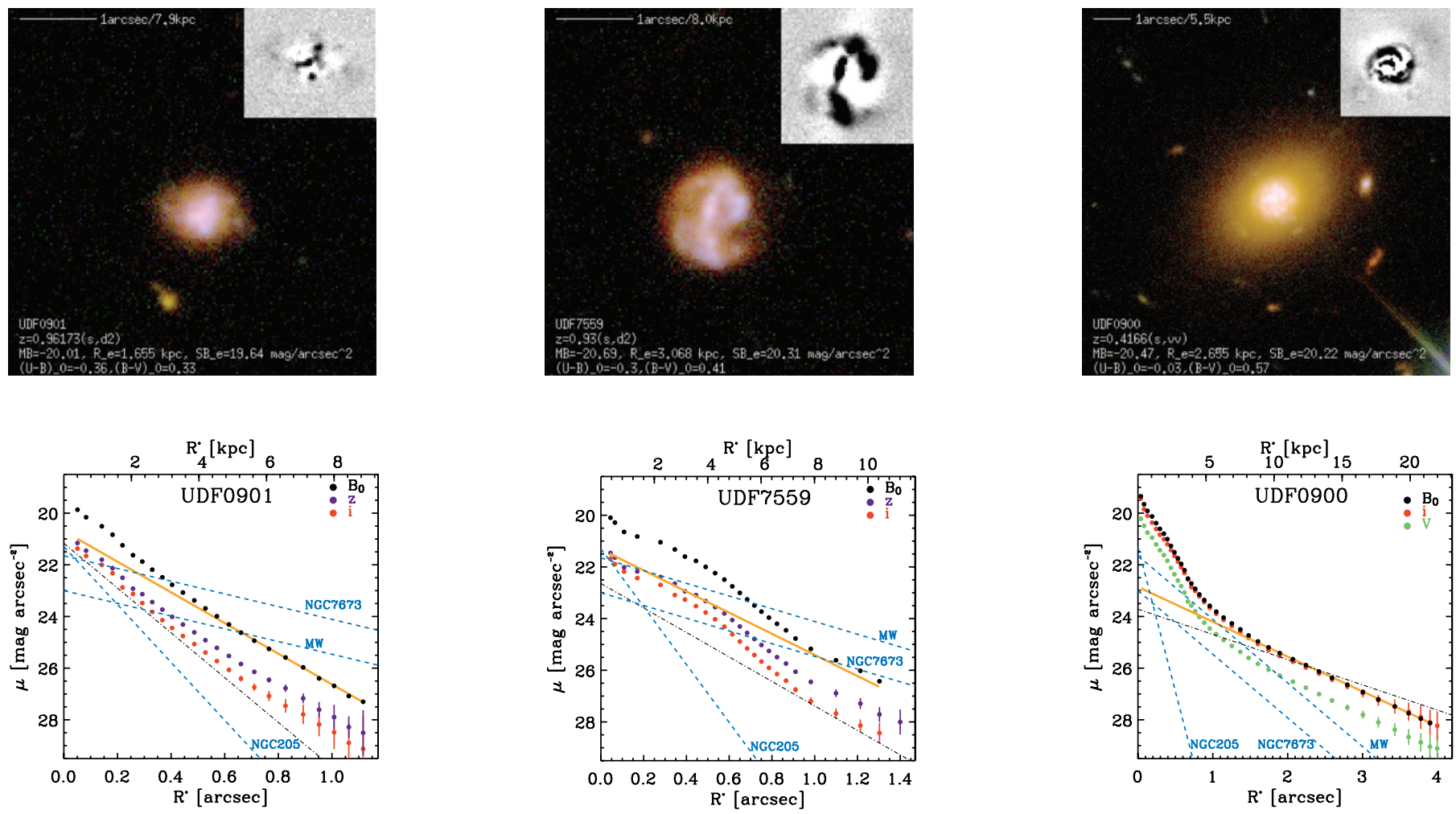

FIG. 1.-Top: Three-color images from HST B, $V$, and $i$, using nonlinear, nonsaturating intensity scaling (Lupton et al. 2004). UDF $901\left(\sigma_{v}=51.6 \mathrm{~km} \mathrm{~s}{ }^{-1}\right)$ and UDF $7559\left(\sigma_{v}=109.5 \mathrm{~km} \mathrm{~s}^{-1}\right)$ are examples of typical LCBGs—compact, narrow emission line galaxies (CNELGs) and more extended, broader line objects. UDF 900 is one of two extended, low surface brightness galaxies with a bright, compact nucleus. The insets (gray) show small-scale $i$-band residuals after subtracting smooth GALFIT models. The spatial scaling is equal to that of the three-color images. Bottom: Surface brightness profiles. Colored dots $(V, i, z)$ show the observed profiles closest in wavelength to the rest-frame $B$ band. Black dots $\left(B_{0}\right)$ denote the rest-frame $B_{\text {vega }}$ profile, $k$-corrected and corrected for cosmological surface brightness dimming. The dashed blue lines show the Milky Way (MW) disk, an LSB disk in a nearby LCBG (NGC 7673), and the dE galaxy NGC 205 (Choi et al. 2002) in rest-frame $B$, for comparison. Note the surface brightness limits of the rest-frame $B$ SBPs $\left(>26\right.$ mag arcsec $^{-2}$ ) and the detectability of large disks at redshifts $z \sim 1$. Thick orange lines show fits to the extended exponential components in rest-frame $B$ LAZY profiles. Dot-dashed lines give the extended exponential component yielded by GALFIT decompositions in the $i$ band, to illustrate PSF effects on LAZY-derived profiles.

Deep Field (UDF) ${ }^{1}$ to search for extended disk components in 26 LCBGs. Details of this study can be found in a forthcoming paper (K. G. Noeske et al. 2006, in preparation, hereafter Paper II). Throughout this Letter, we adopt a $\Lambda$ CDM cosmology $\left(H_{0}=70 \mathrm{~km} \mathrm{~s}^{-1} \mathrm{Mpc}^{-1}, \Omega_{M}=0.3, \Omega_{\Lambda}=0.7\right)$.

\section{SAMPLE SELECTION AND DATA PROCESSING}

We adopted similar rest-frame selection criteria to those of Garland et al. (2004), Werk et al. (2004), and Hoyos et al. (2004) for local LCBG samples: (1) blue rest-frame $B-V \leq 0.6$, (2) average rest-frame surface brightness within the half-light radius $\mu_{e} \leq 21 B$ mag $\operatorname{arcsec}^{-2}$, (3) $M_{B} \leq-18.5$, and (4) half-light radius $r_{e} \leq 3.5 \mathrm{kpc}$. These somewhat arbitrary limits include both the extremely compact CNELGs and larger LCBGs, more comparable to those analyzed by, for example, Phillips et al. (1997). The galaxies were selected from the UDF SExtractor catalog (S. Beckwith et al. 2006, in preparation), after computing linear extents and rest-frame photometry, using the DEEP2 $k$-corrections (Willmer et al. 2005) and robust spectroscopic (Szokoly et al. 2004; Le Fèvre et al. 2004; Vanzella et al. 2005; D. C. Koo et al. 2006, in preparation) and photometric (Wolf et al. 2004) redshifts. After removing doubtful cases and two active

${ }^{1}$ Comprising 56, 56, 144, and 144 orbits of integration time in the $B$ (F435W), $V$ (F606V), $i$ (F775W), and $z$ (F850LP) filters, respectively; PI S. Beckwith, STScI. galactic nuclei, this yielded 26 objects at $0.21<z<1.25$, about two-thirds of them at $z>0.9$. See Figure 1 for examples.

All LCBG images were analyzed through two surface photometry methods: (1) One-dimensional surface brightness profiles (SBPs) were derived using the morphology-adaptive mask algorithm "LAZY" described in Papaderos et al. (2002; "method [iv]") and Noeske et al. (2003), with procedures detailed in those papers. LAZY can treat the irregular morphologies of LCBGs and is robust at low intensity levels, allowing one to detect and measure, or reject, large LSB structures. The resulting SBPs typically showed approximately exponential, moderately extended components (see $\S 3$ ), which we fitted with exponential laws outside the brighter central excesses (see Noeske et al. 2003). (2) Point-spread function (PSF) effects on measured structural parameters of the extended components are nonnegligible. For brighter components with roughly elliptical isophotes, PSF treatment is provided by the GALFIT code (Peng et al. 2002). We decomposed the LCBGs into two exponential components. Obviously unphysical fits due to very irregular morphologies were rejected, as were fitted extended components fainter than an empirical reliability limit of $25 \mathrm{AB}$ mag in $i$ and $z$.

Comparisons between LAZY and GALFIT, and reliability assessments, are detailed in Paper II. Exponential scale lengths $\left(R_{s}\right)$ from LAZY are typically overestimated for small objects $\left(R_{s} \lesssim 1.5 \mathrm{kpc}\right)$ by a factor of $\lesssim 1.3$, few up to $\sim 2$, while for larger scale lengths, GALFIT can underestimate $R_{s}$ by a factor 
$\lesssim 1.3$ (see Fig. 2). The two methods hence bracket the true scale lengths. Example SBPs are shown in Figure 1. In most cases, the rest-frame $B$-band SBPs reached beyond the restframe Holmberg radius ( $26.5 \mathrm{~B}$ mag $\operatorname{arcsec}^{-2}$ ) even for compact objects at $z \sim 1$ (see object UDF 901 in Fig. 1).

\section{RESULTS}

\subsection{LCBGs: Star Formation within More Extended, Evolved Galaxies}

The SBPs of most LCBGs display a moderately extended, roughly exponential component, corresponding to a mostly fairly regular outer component in the images (Fig. 1). At smaller radii the SBPs show brighter, smaller components, in excess of the extended exponentials. These range from bright nuclei to extended emission over a large part of the galaxy and reflect the irregular blue emission seen in the images - that is, probably the ongoing SF. For larger LCBGs, this structure has been reported previously (Koo et al. 1995; Phillips et al. 1997; Guzmán et al. 1998; Hammer et al. 2001). For smaller LCBGs, our current UDF data set verifies what the data of Guzmán et al. (1998) suggested: these objects, similar to local H II galaxies or distant CNELGs, also have roughly exponential stellar components that predate the ongoing SF.

Rest-frame colors of the extended components are $-0.3 \lesssim$ $U-B \lesssim 0.3$ and $0.3 \lessgtr B-V \lesssim 0.9$ for $\sim 90 \%$ of the LCBGs, on average $\sim 0.2 \mathrm{mag}$ redder than the SF excesses. For these colors, simple stellar population models for up to solar metallicity (Anders \& Fritze-von Alvensleben 2003) yield minimum stellar ages of $\gtrsim 100$ Myr to several gigayears. More extended SF histories (e.g., Bicker et al. 2004) lead to higher ages. If extinction is significant in the extended components, outside strong SF, these age limits will decrease.

We will refer to "extended components" rather than "disks," as we lack resolved kinematic data for most objects. Morphologies are often disklike for larger LCBGs (see $\S 1$ ) but ambiguous for small objects, which could be either spheroids or disks.

\subsection{Structure of the Extended Components}

Figure 2 compares the extended components of the LCBGs with samples of nearby disk galaxies and dwarf galaxies with exponential SBPs. The disk sample by Lu (1998) was chosen because of its completeness in local field disk scale lengths, and the UMa cluster sample by Tully et al. (1996) was added to include disks from higher density environments.

It is evident that the extended components of almost all LCBGs have small scale lengths $\left(R_{s} \lesssim 2 \mathrm{kpc}\right)$ : at any given luminosity, they are comparable to the local disk galaxies with the smallest $R_{s}$. This result is robust against uncertainties in $R_{s}$. The less luminous exponential components are also compatible with those of relatively compact, luminous local dwarf galaxies (compact dE, or stellar hosts of blue compact dwarfs). The only two exceptions are UDF 900 (the LCBG with the largest $R_{s}$ in Fig. 2; see also Fig. 1), which shows a large LSB component $\left(\mu_{0, B} \approx 22.9 \mathrm{mag} \operatorname{arcsec}^{-2}, R_{s}=4.4 \mathrm{kpc}\right)$ with an exponential SBP but no spiral features, and a galaxy that may have a truncated disk with a small outer but larger inner scale length (Fig. 2) ${ }^{2}$ Both galaxies have bright central regions that led to their classification as LCBGs.

\footnotetext{
${ }^{2}$ The second-largest LAZY-derived scale length in Fig. 2 is affected by PSF wings from a bright nucleus and is in fact smaller; see the connected GALFIT data point.
}

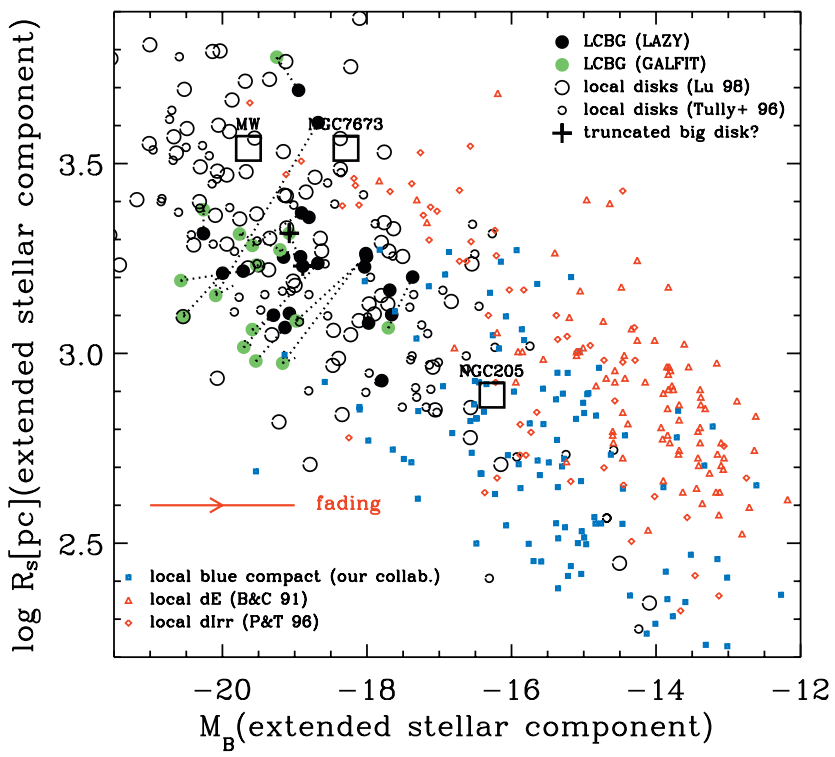

FIG. 2.-Exponential scale length $R_{s}$ vs. absolute $B$-band magnitude for the extended exponential components in LCBGs. Filled black circles denote LAZY, and green ones GALFIT, decompositions (see $\S 2$ ). LAZY scale lengths are inclination corrected, assuming that the extended components are inclined disks, i.e., are upper limits for spheroids. Open circles, local disk galaxy samples from the UMa cluster (Tully et al. 1996) and from field environments in the local supercluster (Lu 1998); blue squares, stellar host galaxies of blue compact dwarfs (Gil de Paz \& Madore 2005 and references therein); red diamonds, dwarf irregular galaxies (Patterson \& Thuan 1996); red triangles, dwarf elliptical galaxies (Binggeli \& Cameron 1991); open boxes, the dwarf elliptical NGC 205 (Choi et al. 2002) and the disks of the Milky Way (3.5 kpc; de Vaucouleurs \& Pence 1978) and the nearby LCBG candidate NGC 7673 (see Pisano et al. 2001; SBP derived in this work, see Paper II). The arrow shows the effect of fading.

\section{DISCUSSION AND CONCLUSIONS}

This structural study of their extended components reveals that $\sim 90 \%$ of LCBGs at $z \sim 0.2-1.3$ truly are small galaxies. For these, suspected large disks with $R_{s}$ similar to the Milky Way, or the extended low surface brightness component found in a local LCBG (NGC 7673), can be ruled out down to surface brightnesses $\gtrsim 26 B$ mag $\operatorname{arcsec}^{-2}$ (cf. Fig. 1). The discovery of one or possibly two LCBGs $(\$ 10 \%)$ that are large, disklike galaxies with bright nuclei supports the scenario of some LCBGs at higher $z$ being nuclear starbursts, or possibly connected to bulge formation, in large disks.

The available scale lengths help to constrain the passive, post-starburst evolution scenario for LCBGs. Fading of the smaller, brighter SF component will affect the overall photometric structure of an LCBG. The older extended components alone will, however, likely fade more homogeneously and thus largely maintain their scale lengths. The extended components could fade by several magnitudes, the bluest in principle up to $5 B$ mag from $z=1$ to $z=0$ if they were $\sim 100$ Myr old simple stellar populations (see above). However, the scale lengths suggest that LCBGs evolve into local small disks, or different types of larger dwarf and low-mass galaxies, assuming that their subsequent evolution does not involve strong disk growth. ${ }^{3}$

${ }^{3}$ For low-mass LCBGs similar to blue compact dwarf galaxies, energy input from strong SF could also affect their stellar mass distribution (Papaderos et al. 1996). 
Subsequent evolution into local large disks of at least the Milky Way scale length would require an LCBG's extended stellar component to grow substantially, by a factor $\gtrsim 1.5$, and typically greater than 2 . The data cannot exclude, but neither do they evidence, such ongoing growth: For larger LCBGs with a disklike morphology, we find no evidence of simple inside-out growth, such as blueing at large radii. For these galaxies, local disks with small scale lengths provide a possible descendant population, so that the substantial disk growth that Hammer et al. $(2001,2005)$ propose for similar and somewhat larger LCBGs may not be required. ${ }^{4}$ From their line widths, sizes, and stellar masses (Guzmán et al. 2003), disklike LCBGs could well be LCBG phases of local intermediate-mass $\left(M_{*}\right.$ $\sim 10^{10} M_{\odot}$ ) disk galaxies, in agreement with the finding that such galaxies experienced substantial SF since $z \sim 1$ (Heavens et al. 2004; Bell et al. 2005; Hammer et al. 2005). Around small, H II galaxy-like LCBGs, we do not find signs of forming or preexisting large disks. Their morphologies and sizes make these galaxies candidate progenitors of small, low-mass galaxies in the local universe, such as low-mass spheroidal or irregular galaxies. Resolved kinematic data will be important to constrain such scenarios.

We finally note that the blue, irregular emission in most LCBGs that are not large disks extends out to $\gtrsim 1.5-2$ scale lengths of the extended component (Fig. 1, insets). This frac-

\footnotetext{
${ }^{4}$ Note that the selection criteria of Hammer et al. and this work are not fully comparable; their LCBG criteria may favor progenitors of larger local galaxies than our sample.
}

tional extent is similar to nearby LCBGs (Papaderos et al. 1996), where this blue emission is the locus of the ongoing SF and nebular emission. It appears plausible that irregular emission also traces nebular emission in distant LCBGs. If so, then nebular emission will sample a sufficient fraction of their velocity field to provide valid mass estimates: Pisano et al. (2001) showed that nebular emission-line kinematics in nearby LCBGs traces neutral gas kinematics with moderate correction factors ( 0.7). This lends support to distant LCBGs' being mostly lowto intermediate-mass galaxies.

In summary, most LCBGs at intermediate $z$ show brighter, irregular, likely star-forming emission within more extended, regular components with approximately exponential intensity distributions and minimum stellar ages $\gtrsim 100$ Myr. Most extended components have scale lengths that are smaller by factors of more than 1.5-2 than local large disks such as the Milky Way, while one or two of 26 LCBGs are larger, disklike galaxies with bright nuclei. This suggests that $\sim 90 \%$ of LCBGs are progenitors of small disk, irregular, or low-mass spheroidal galaxies in the local universe; $\sim 10 \%$ may represent bulge formation within massive disks.

We wish to thank Professor M. Bershady for the WIYN $R$-band image of NGC 7673. Research by D. C. K. and K. G. N. was partly funded by NSF grants AST 00-71198 and AST 05-07483 and HST grants GO-09126.02-A and AR10321.02-A. We thank the referees for their valuable comments on this Letter, and B. Holden for helpful advice on ACS PSF peculiarities.

\section{REFERENCES}

Anders, P., \& Fritze-von Alvensleben, U. 2003, A\&A, 401, 1063

Barton, E. J. \& van Zee, L. 2001, ApJ, 550, L35

Bell, E. F., et al. 2005, ApJ, 625, 23

Bicker, J., Fritze-von Alvensleben, U., Möller, C. S., \& Fricke, K. J. 2004, A\&A, 413, 37

Binggeli, B., \& Cameron, L. M. 1991, A\&A, 252, 27

Choi, P. I., Guhathakurta, P., \& Johnston, K. V. 2002, AJ, 124, 310

de Vaucouleurs, G., \& Pence, W. D. 1978, AJ, 83, 1163

Faber, S. M., et al. 2005, ApJ, submitted (astro-ph/0506044)

Garland, C. A., Pisano, D. J., Williams, J. P., Guzmán, R., \& Castander, F. J. 2004, ApJ, 615, 689

Gil de Paz, A., \& Madore, B. F. 2005, ApJS, 156, 345

Guzmán, R., Gallego, J., Koo, D. C., Phillips, A. C., Lowenthal, J. D., Faber, S. M., Illingworth, G. D., \& Vogt, N. P. 1997, ApJ, 489, 559

Guzmán, R., Jangren, A., Koo, D. C., Bershady, M. A., \& Simard, L. 1998, ApJ, 495, L13

Guzmán, R., Koo, D. C., Faber, S. M., Illingworth, G. D., Takamiya, M., Kron, R. G., \& Bershady, M. A. 1996, ApJ, 460, L5

Guzmán, R., Östlin, G., Kunth, D., Bershady, M. A., Koo, D. C., \& Pahre, M. A. 2003, ApJ, 586, L45

Hammer, F., Flores, H., Elbaz, D., Zheng, X.-Z., Liang, Y.-C., \& Cesarsky, C. 2005 , A\&A, 430, 115

Hammer, F., Gruel, N., Thuan, T. X., Flores, H., \& Infante, L. 2001, ApJ, 550, 570

Heavens, A., Panter, B., Jimenez, R., \& Dunlop, J. 2004, Nature, 428, 625

Hoyos, C., Guzmán, R., Bershady, M. A., Koo, D. C., \& Díaz, A. I. 2004, AJ, 128, 1541

Koo, D. C., Bershady, M. A., Wirth, G. D., Stanford, S. A., \& Majewski, S. R. 1994, ApJ, 427, L9
Koo, D. C., Guzmán, R., Faber, S. M., Illingworth, G. D., Bershady, M. A., Kron, R. G., \& Takamiya, M. 1995, ApJ, 440, L49

Le Fèvre, O., et al. 2004, A\&A, 428, 1043

Lu, N. Y. 1998, ApJ, 506, 673

Lupton, R., Blanton, M. R., Fekete, G., Hogg, D. W., O’Mullane, W., Szalay, A., \& Wherry, N. 2004, PASP, 116, 133

Madau, P., Ferguson, H. C., Dickinson, M. E., Giavalisco, M., Steidel, C. C., \& Fruchter, A. 1996, MNRAS, 283, 1388

Noeske, K. G., Papaderos, P., Cairós, L. M., \& Fricke, K. J. 2003, A\&A, 410, 481

Papaderos, P., Izotov, Yu. I., Thuan, T. X., Noeske, K. G., Fricke, K. J., Guseva, N. G., \& Green, R. F. 2002, A\&A, 393, 461

Papaderos, P., Loose, H.-H., Fricke, K. J., \& Thuan, T. X. 1996, A\&A, 314, 59

Patterson, R. J., \& Thuan, T. X. 1996, ApJS, 107, 103

Peng, C. Y., Ho, L. C., Impey, C. D., \& Rix, H.-W. 2002, AJ, 124, 266

Phillips, A. C., et al. 1997, ApJ, 489, 543

Pisano, D. J., Kobulnicky, H. A., Guzmán, R., Gallego, J., \& Bershady, M. A. 2001, AJ, 122, 1194

Szokoly, G. P., et al. 2004, ApJS, 155, 271

Tully, R. B., Verheijen, M. A. W., Pierce, M. J., Huang, J.-S., \& Wainscoat, R. J. 1996, AJ, 112, 2471

Vanzella, E., et al. 2005, A\&A, 434, 53

Werk, J. K., Jangren, A., \& Salzer, J. J. 2004, ApJ, 617, 1004

Willmer, C. N. A., et al. 2005, ApJ, submitted (astro-ph/0506041)

Wolf, C., et al. 2004, A\&A, 421, 913 